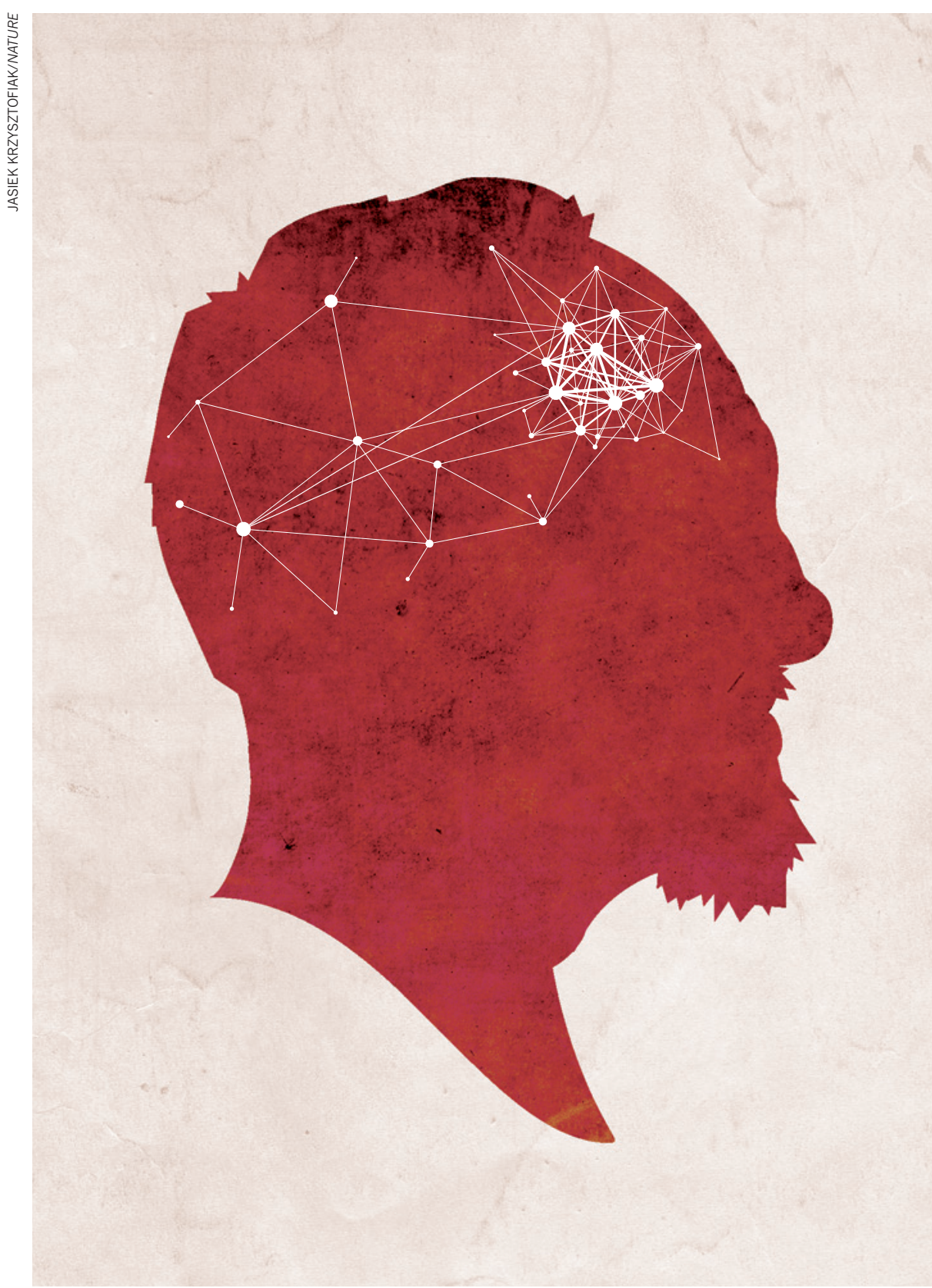

\section{Study neuron networks to tackle Alzheimer's}

Researchers have generally looked for signs of Alzheimer's disease in the whole brain or at the singlegene level. The biggest clues will come from monitoring collections of neurons, says Kenneth S. Kosik.
$\mathrm{T}$ wenty years of research and more than US\$1-billion worth of clinical trials have failed to yield an effective drug treatment for Alzheimer's disease. Most neuroscientists, clinicians and drug developers now agree that people at risk of the condition will probably need to receive medication before the onset of any cognitive symptoms. Yet a major stumbling block for early intervention is the absence of tools that can reveal the first expression of the insidious disease.

So far, researchers have tended to focus on macroscopic changes associated with the disease, such as the build up of insoluble plaques of protein in certain areas of the brain, or on individual genes or molecular pathways that seem to be involved in disease progression.

I contend that detecting the first disruptions to brain circuitry, and tracking the anatomical and physiological damage underlying the steady cognitive decline that is symptomatic of Alzheimer's, will require tools that operate at the 'mesoscopic' scale: techniques that probe the activity of thousands or millions of networked neurons. Although such tools are yet to be realized, several existing technologies indicate that they are within reach.

\section{CHARTED TERRITORY}

All the current approaches that are used to diagnose Alzheimer's are crude and unreliable. Take the classic biomarkers of the disease: a build up of plaques of the protein $\beta$-amyloid in a person's cerebral cortex, for instance, or elevated levels of the tau protein and dampened levels of $\beta$-amyloid in their cerebrospinal fluid. Although such markers are predictive of the disease, the interval between their appearance and the onset of cognitive problems is hugely variable, ranging from months to decades.

Frequently, people with a high density of plaques or an ominous cerebrospinal fluid reading show no signs of dementia in behavioural tests. Others show the classic symptoms of the disease, such as memory loss, confusion and inability to formulate a simple plan.

More consistently linked to cognitive difficulties are neurofibrillary tangles aggregates of tau protein commonly found in the hippocampus and amygdala (the parts of the brain involved in memory and emotion) of people with Alzheimer's, and in the cerebral cortex. Yet even this correlation is imperfect. And unlike for amyloid plaques, which can be monitored using a scanning technique known as positron

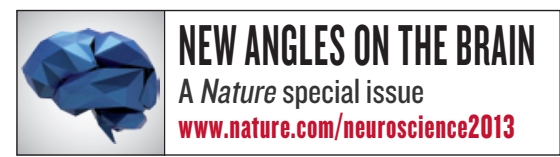


emission tomography (PET), no imaging procedure is yet available to detect tangles in living people.

So although all these markers can be harbingers of disease, none of them captures the subtle disruptions to brain circuitry that mark the onset of cognitive decline or enable researchers to track disease progression from week to week and month to month. Nor do they reveal anything about the even shorter-term fluctuations in cognitive capacity frequently observed in people with Alzheimer's. Relatives and carers often report, for instance, that for brief periods, a person with Alzheimer's might behave normally and be able to engage in conversation, and then an hour later be unable to remember what just transpired.

Aside from biomarkers, clinicians use reams of neuropsychological tests to diagnose Alzheimer's disease. These test a person's memory, emotional responses, language skills, and ability to solve problems or count. However, such tests fail to capture the subtleties of the cognitive deficits at every stage of the disease. For example, asking someone to recall a recently viewed list of items will not reveal how well they are able to pull together memory fragments to make appropriate decisions and predictions. Similarly, being able to join up sequentially numbered dots (a standard test of executive function) is a far cry from mentally mapping out the steps needed to achieve some purpose in daily life, such as food shopping.

\section{IMAGING ISSUES}

One of the various types of brain imaging commonly used to probe and diagnose Alzheimer's is functional magnetic resonance imaging (fMRI). This tracks changes to blood oxygenation in the brain while a person is resting or doing certain cognitive tasks. The aim is to reveal faulty wiring or differences in regional brain activity from what is usually observed. However, standard fMRI is limited to scanning the 86 billion human brain neurons and several thousand times more synapses with fewer than 20,000 voxels (volumetric pixels). With this technique, each voxel corresponds to an arbitrary volume of tissue rather than to a specific brain network that mediates cognitive processes. PET, which assesses blood flow and metabolism, as well as the location and density of amyloid plaques, has an even lower spatial resolution.

Furthermore, the current tools used to diagnose Alzheimer's disease and track its progression do not account for innate or acquired individual differences in brain structure that can drastically alter people's tolerance of brain pathology.

Numerous studies have suggested, for instance, that education and higher intellectual achievement, such as greater proficiency in languages, writing and speaking, can protect people from clinical Alzheimer's disease (with which people show severe cognitive deficits in behavioural tests). For example, a long-running study of nuns, which began in 1986, found that those diagnosed with the disease through post-mortem examinations had, decades before their death, displayed inferior language skills in application letters sent to convents compared with those without the disease ${ }^{1}$. This may help to explain why people with similar amyloid burdens in their cerebral cortex can show such dramatic differences in cognitive performance ${ }^{2}$. It also highlights why researchers should avoid evaluating the efficacy of a drug on its ability to break down amyloid plaques alone.

\section{UNCHARTED TERRITORY}

Several lines of evidence suggest that networks of neurons in the brain operate as local processing units, with few long-range connections between them - an organizational pattern known as small world ${ }^{3}$. This evidence includes partial wir"Researchers ing diagrams of the should avoid fly and mammalian evaluating brain obtained from the efficacy anatomical tracings, of adrug on circuit physiology its ability to and by reconstructbreakdown ing nanometre-thick amyloid slices of brain tissue. plaques Optical-imaging techalone." niques used to track neural activity across the entire brain in organisms such as zebrafish larvae also support this idea ${ }^{4}$.

I believe that tools to analyse how neuron networks operate in the human brain will be crucial to probing the changes to brain circuitry underlying cognitive impairment in Alzheimer's disease. One possibility is using minimally invasive 'nanosensors' that can travel to the brain through blood vessels and communicate neural activity ${ }^{5}$. Diamond-based materials are currently being developed to track the movements of single electrons across nerve membranes.

Even now, several techniques could offer preliminary insights about the collective workings of different regions of the human brain. For example, particularly powerful MRI systems can scan the entire living human brain at a resolution of about 0.7 cubic millimetres (most are limited to $1-\mathrm{mm}^{3}$ resolution $)^{6}$.

Meanwhile, researchers have used another imaging technique known as magnetoencephalography (MEG) - which maps neural activity by recording the magnetic fields produced by electrical currents in the brain - to predict the development of preclinical Alzheimer's disease, known as mild cognitive impairment, in 5 out

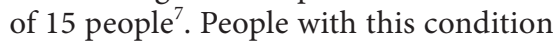

experience difficulties that are noticeable to them and to others but that are not severe enough to interfere with daily life. MEG has a poorer spatial resolution than MRI, but records responses in milliseconds rather than in seconds or minutes, capturing more of the numerous intermediary cognitive steps involved in mediating a person's response to a picture, word or task.

Likewise, molecular analyses could shed light on brain activity at the mesoscopic scale. Sequencing the RNA molecules expressed in different regions of the brain, in tissues taken from people who have died, has revealed coregulated networks of genes associated with late-onset Alzheimer's ${ }^{8}$. Of enormous value would be detailed maps showing the genes expressed, where they are expressed and how they relate to neural networks - as have been obtained for the sea urchin ${ }^{9}$.

\section{LOOKING AHEAD}

Such mesoscopic-scale analyses are precisely what the US BRAIN (Brain Research through Advancing Innovative Neurotechnologies) Initiative is about. Announced by US President Barack Obama in April, the aim of this project is to map and monitor the neural connections in the entire brains of experimental animals, and ultimately in the human cerebral cortex.

Several influential neuroscientists have questioned whether an understanding of consciousness, perception, imagination, emotion and abstraction will emerge from measuring brain activity at the mesoscopic scale. I am similarly sceptical of such lofty claims. Yet I applaud the BRAIN Initiative for its potential to advance Alzheimer's disease research. Monitoring neuron networks is exactly what neuroscientists need to better understand, and ultimately to treat, a disease that is now costing the United States alone upwards of $\$ 200$ billion every year.

Kenneth S. Kosik is at the Neuroscience Research Institute, and professor in the Department of Cellular, Molecular and Developmental Biology at the University of California, Santa Barbara, California 93106, USA.

e-mail:kenneth.kosik@lifesci.ucsb.edu

1. lacono, D. et al. Neurology 73, 665-673 (2009).

2. Rowe, C. C. et al. Neurobiol. Aging 31, 1275-1283 (2010).

3. Bullmore, E. \& Sporns, O. Nature Rev. Neurosci. 13, 336-349 (2012).

4. Ahrens, M. B. et al. Nature 485, 471-477 (2012).

5. Seo, D., Carmena, J. M., Rabaey, J. M., Alon, E. \& Maharbiz, M. M. Preprint available at http://arxiv. org/abs/1307.2196 (2013).

6. van Rooden. S. et al. Alzheimers Dement. http:// dx.doi.org/10.1016/j.jalz.2013.02.002 (2013).

7. Zamrini, E. et al. Int. J. Alzheimers Dis. 2011, 280289 (2011)

8. Lambert J.-C. et al. Nature Genet. http://dx.doi. org/10.1038/ng.2802 (2013).

9. Peter, I. S., Faure, E. \& Davidson, E. H. Proc. Nat Acad. Sci. USA 109, 16434-16442 (2012). 